\title{
Phenotypic detection of carbapenem resistant Enterobacteriaceae and characterization of Klebsiella pneumoniae carbapenemase (KPC) producing Klebsiella pneumoniae
}

\author{
Abidemi Esther Ojo ${ }^{* 1}$, Seun Owolabi Adebajo ${ }^{1}$, Kolapo A Fasina ${ }^{2}$, Bolutife Odejinmi ${ }^{1}$, \\ Olugbenga Anthony Ojo ${ }^{3}$, Abiola Oladotun ${ }^{1}$, Joshua Ibeaha ${ }^{1}$ \\ 1. Microbiology Department, College of Biosciences, Federal University of Agriculture, PMB 2240, Abeokuta, Ogun State, Nigeria. . \\ 2. Biotechnology Division, Rubber Research Institute of Nigeria, PMB 1049, Iyanomo, Benin City, Edo State, Nigeria. \\ 3. Department of Pharmacognosy, Faculty of Pharmacy, Madonna University, Port Harcourt, Rivers State, Nigeria.
}

\section{A R T I C L E I N F O}

\section{Article history:}

Received 23 June 2020

Received in revised form 7 August 2020

Accepted 8 August 2020

\section{Keywords:}

Carbapenem resistant Enterobactereaceae

Modified Hodge test

Boronic acid-based inhibition test

Klebsiella pneumoniae carbapenemase

\begin{abstract}
A B S T R A C T
Background: Carbapenem-resistant Enterobacteriaceae (CRE) pose a great threat to the public health sector as a result of its high communicability, high morbidity and mortality rates. This study investigated the occurrence of carbapenem resistant Enterobacteriaceae and the prevalence of Klebsiella pneumoniae carbapenemase (KPC) producing Klebsiella pneumoniae as a mechanism of resistance. Method: One hundred and ten Enterobacteriaceae isolates were cultured from different samples of patients in Intensive Care Units of two hospitals. Using Clinical and Laboratory Standards Institute (CLSI) Guidelines of 2017. Carbapenemase production was determined phenotypically using Brilliance CRE agar, Carbapenem inactivation method and confirmed with Modified Hodge test (MHT). Modified Hodge test positive isolates were screened for KPC-producing Klebsiella pneumoniae using boronic acid-based inhibition test. Result: Phenotypic results revealed an occurrence of $10.9 \%$ (12/110) isolates for carbapenem resistant Enterobacteriaceae while KPC-producing Klebsiella pneumoniae prevalence was $3.6 \%$ contributing $33.3 \%$ to the burden of carbapenem production. The occurrence of carbapenem-resistant Enterobacteriaceae in this study was relatively high and KPCKlebsiella pneumoniae had a significant contribution to the burden of CRE. Conclusion: Hence, a sinewy antibiotic stewardship is needed in this regard.
\end{abstract}

\section{Introduction}

Carbapenem-resistant Enterobacteriaceae (CRE) are highly pathogenic and antibiotic-resistant Gram negative organisms producing nosocomial infections because of its innate capacity for fast transmission within healthcare facilities and its environs [1]. Carbapenemases are beta-lactamase enzymes that inactivate carbapenems, and other betalactam antibiotics [2]. Carbapenem-resistant Enterobacteriaceae pose a great threat to the public health sector due to its high rate of transmission which causes high morbidity and mortality coupled with very minute chances of treatment $[3,4]$.
Currently, hospitals remain the major sites of transmission for CRE infections. Poor hygienic practices remains the largest cause of CRE transmission and includes insufficient sterilization of drug shelves, contact points in wards, and movable health care instruments, such as X-ray and ultrasound machines useful for both CRE and non-CRE patients [5]. Carbapenemase-producing Enterobacteriaceae (CPE) are predominant in the clinical isolates of CRE where there is high burden of CRE [6]. The difference between CRE and CPE is very simple and clear. Majority (with the exception of very few) of 
Enterobacteriaceae that are resistant to carbapanems produce carbapenemase (that is, there are some CRE with a carbapenem resistance mechanism apart from carbapenemase production). Likewise, most but not all CPE, exhibit resistance to carbapenems (i.e. some display minimal carbapenem MICs and remain phenotypically irresistible to carbapenems) [1].

In line with Ambler classification system, classes which require serine at their active site, are classified as A and D. Class A serine $\beta$-lactamases family (e.g. KPC-type enzymes) consist of the Klebsiella pneumoniae carbapenemase (KPC), IMI, SME and GES enzymes. The group which requires zinc at their active site is classified as B, and this latter class is also referred to as Metallo- $\beta$-lactamases (MBLs). This includes the IMP, VIM, NDM, and SPM enzymes. The class D serine $\beta$-lactamases (OXA), most notable of which is the OXA-48 enzyme, which hydrolyzes oxacillin, provide a good example of the variety of mechanisms that can be used to transfer resistance [7,8]. Each of these classes have their peculiar functional characteristics in relation to their phenotypic observation and hospital care. Boronic acid compounds are known as the best inhibitors of class A carbapenemases and this includes the KPC. Hence, these inhibitors can be employed in their detection [9]. The blaOXA genes which encode OXA $\beta$-lactamases are found on both chromosomes and plasmids and they have their natural reservoir in environmental bacteria and deepsea microflora.

Based on the foregoing, this study aimed at determining the occurrence of CRE and clinical significance of KPC producing Klebsiella pneumoniae in Ogun State, Nigeria.

\section{Materials and Methods}

\section{Study area}

The study was conducted at Sacred Heart Hospital and Federal Medical Centre both in Abeokuta, Ogun State, Nigeria.

\section{Ethical considerations}

This study was approved by the Federal University of Agriculture, Abeokuta, Nigeria through the Department of Microbiology and presented to the two concerned health review boards for ethical approval. Ethical clearance was obtained from the institutional Review Boards of Federal Medical Centre, Idi Aba, Abeokuta and Sacred Heart Hospital, Lantoro, Abeokuta, the two hospitals involved in this study who ensured that the study was performed in compliance with the 'The Code of Ethics of the World Medical Association'.

\section{Selection and description of participants}

The sampled populations were patients on acute care having spent minimum of 3 days on admission in different wards, with most of them having recently undergone surgical operations. The patient must have spent minimum of 3 days on admission in the same hospital as an inclusion criterion in this study. The patients had various disease conditions including urinary tract infections (UTIs), gastrointestinal disorders, diabetes, acute nephritis and jaundice. A total of 170 samples (including cerebrospinal fluid, stool and urine obtained through catheter) were obtained between July 2019 and February 2020, and transported to the laboratory according to standard methods [10].

\section{Bacterial identification and characterization}

Samples were inoculated on Brilliance CRE agar (Oxoid, UK), Blood agar (Oxoid, UK) and MacConkey agar (Oxoid, UK) and incubated at $37^{\circ} \mathrm{C}$ for $24 \mathrm{~h}$ aerobically. Typical color appearance on Brilliance CRE agar, colonial morphology and attributes on other agars were observed and distinct colonies were Gram stained in line with standard methods [10]. Gram negative rods were subjected to further biochemical tests.

All Gram negative rods were further confirmed using the Microbact Gram-negative identification system ${ }^{\mathrm{TM}}$ (Oxoid, UK) 24E according to the manufacturer's instructions.

\section{Carbapenem inactivation method (CIM)}

Carbapenem-resistant Enterobacteriaceae isolates from Brilliance CRE agar were subjected to CIM test. With the CIM, a 10- $\mu$ g meropenem disk was first incubated in a suspension of the test strain at $37^{\circ} \mathrm{C}$ for 2 hours. After incubation, the meropenem disk was removed from the solution and placed on a Mueller Hinton agar plate inoculated with susceptible E. coli ATCC 25922 as with the Modified Hodge test (MHT). Carbapenemase production was indicated by the inactivation of meropenem, thus allowing for uninhibited growth of $E$. coli. If no carbapenemase is produced, a clear inhibition zone was observed.

\section{Phenotypic carbapenem detection}

Carbapenem-resistant Enterobacteriaceae isolates from Brilliance CRE agar were further subjected to MHT test for confirmation of carbapenem production. Modified Hodge test (considered the gold standard), carbapenem inactivation method and boronic acid inhibition test were employed in 
carbapenem production detection. In the MHT, a tenth dilution of $0.5 \mathrm{McF}$ arland suspension of $E$. coli ATCC 25922 was used to evenly inoculate a Mueller-Hinton agar plate, and a $10-\mu \mathrm{g}$ imipenem disk (Oxoid, UK) placed in the center of the plate. The suspected CPE strain was then streaked from the edge of the disk to the edge of the plate to form a straight line of thick inoculum and incubated for 24 hrs. The growth of susceptible $E$. coli in the background towards the disk, creating a cloverleaflike appearance indicated carbapenemase production by the test isolate according to CLSI [11]. Quality control was performed with control strains using MHT positive Klebsiella pneumoniae ATCC BAA1705 and for negative control Klebsiella pneumoniae ATCC 700603.

\section{Boronic acid inhibition test}

A $350 \mu \mathrm{g}$ of 2-aminophenyl boronic acid was applied to an ertapenem or meropenem disk. An increase in the zone of inhibition of $\geq 5 \mathrm{~mm}$ compared to the ertapenem or meropenem disk alone (control) indicated KPC production after incubation for $24 \mathrm{hrs}$ at $37^{\circ} \mathrm{C}$.

\section{Data Analysis}

Data analysis was carried out using Descriptive Statistics in Statistical Analysis software (SAS).

\section{Results}

From the 170 clinical isolates collected in this study, only 110 belonged to the family Enterobacteriaceae. These 110 isolates were tested for carbapenem production and only 12 (11.0\%) exhibited carbapenem production using MHT as the gold standard. Brilliance CRE agar also revealed 12 isolates were positive for carbapenem production while CIM had 11 positive isolates for carbapenem production (Table 1).

From the 110 Enterobacteriaceae, 52.7\% (58) were Klebsiella pneumoniae, followed by E. coli 38 (35.5\%) and Proteus mirabilis 2(1.8\%). Proteus vulgaris and Pseudomonas aeruginosa were $1(0.9 \%)$ and $10(9.1 \%)$ respectively (Table 1). Out of the 110 screened for carbapenem production, 56 were from patients that had gastroenteritis from which five (4.6\%) produced carbapenem (2 Klebsiella pneumoniae, 2 E. coli and 1 Pseudomonas aeruginosa). While none of the 2 isolates from acute nephritis and 1 from diabetes mellitus also known as sugar diabetes was negative for MHT. These isolates from acute nephritis and sugar diabetes didn't not show any resistance to carpabenem, hence were not amongst the isolates further studied in this research.

Only $7(6.4 \%)$ out of the fifty-one UTIs isolates were MHT positive. Note that there was no growth from CSF samples cultured (Table 2).

More (4/5) of the KPC-Enterobactereaceae from this present study were from Klebsiella pneumoniae followed by $E$. coli (1/5) shown in table (3) below.

The most isolated organism in this study was Klebsiella pneumoniae and it was mostly isolated in patients with UTIs (Table 3). From the total 110 Enterobacteriaceae, Klebsiella pneumoniae was $58(52.7 \%)$ out of which seven $(12.1 \%)$ were positive for carbapenemase production. Five (41.6\%) isolates, consisting of $4(33.3 \%)$ Klebsiella pneumoniae and $1(8.3 \%)$ E. coli isolates, out of the seven were positive for KPC by boronic acid-based inhibition test.

Table 1. Percentage frequency of detection of CRE in three phenotypic methods.

\begin{tabular}{|c|c|c|c|c|}
\hline Isolates & Total Screened (\%) & MHT +ve (\%) & $\begin{array}{c}\text { Brilliance CRE agar } \\
\text { +ve }(\%)\end{array}$ & CIM +ve (\%) \\
\hline Klebsiella pneumoniae & $58(52.7)$ & $7(6.4)$ & $7(6.4)$ & $6(7.3)$ \\
\hline E. coli & $38(35.5)$ & $4(3.6)$ & $4(3.6)$ & $4(3.6)$ \\
\hline Proteus mirabilis & $2(1.8)$ & $0(0)$ & $0(0)$ & $0(0)$ \\
\hline Proteus vulgaris & $1(0.9)$ & $0(0)$ & $0(0)$ & $0(0)$ \\
\hline Pseudomonas aeruginosa & $10(9.1)$ & $1(0)$ & $1(0)$ & $1(0)$ \\
\hline Total & 110 & $12(10.9)$ & $12(10.9)$ & $11(11.8)$ \\
\hline
\end{tabular}


Table 2. Incidence of CRE in different isolates in relation to different disease condition.

\begin{tabular}{|l|c|c|c|c|c|c|}
\hline \multicolumn{1}{|c|}{ Isolates } & $\begin{array}{c}\text { Gastroenteritis } \\
\text { (CRE) }\end{array}$ & $\begin{array}{c}\text { Acute nephritis } \\
\text { (CRE) }\end{array}$ & $\begin{array}{c}\text { UTI } \\
\text { (CRE) }\end{array}$ & $\begin{array}{c}\text { Diabetes } \\
\text { (CRE) }\end{array}$ & $\begin{array}{c}\text { CSF } \\
\text { (CRE) }\end{array}$ & Total \\
\hline Klebsiella pneumoniae & $31(2)$ & $1(0)$ & $26(5)$ & $0(0)$ & $0(0)$ & $58(7)$ \\
\hline E. coli & $18(2)$ & $0(0)$ & $20(2)$ & $0(0)$ & $0(0)$ & $38(4)$ \\
\hline Proteus mirabilis & $2(0)$ & $1(0)$ & $0(0)$ & $0(0)$ & $0(0)$ & $3(0)$ \\
\hline Proteus vulgaris & $1(0)$ & $0(0)$ & $0(0)$ & $0(0)$ & $0(0)$ & $1(0)$ \\
\hline Pseudomonas aeruginosa & $4(1)$ & $0(0)$ & $5(0)$ & $1(0)$ & $0(0)$ & $10(1)$ \\
\hline & $56(5)$ & $2(0)$ & $51(7)$ & $1(0)$ & $0(0)$ & $110(12)$ \\
\hline
\end{tabular}

Table 3. Percentage frequency of KPC - Enterobacteriaceae.

\begin{tabular}{|l|c|c|}
\hline \multicolumn{1}{|c|}{ Isolates } & Frequency of MHT+VE & Frequency of BAI test +ve (\%) \\
\hline Klebsiella pneumoniae & 7 & $4(33.3)$ \\
\hline E. coli & 4 & $1(8.3)$ \\
\hline Proteus mirabilis & 0 & $0(0)$ \\
\hline Proteus vulgaris & 0 & $0(0)$ \\
\hline Pseudomonas aeruginosa & 1 & $0(0)$ \\
\hline Total & 12 & $5(41.6)$ \\
\hline
\end{tabular}

\section{Discussion}

Global CRE infection rates have been reported to range between zero and $89 \%$, but mostly between $7.6-44.4 \%$. This broad range might be attributed to varied populations studied [12]. This present study is not exceptional by revealing a prevalence of $10.9 \%$ carbapenem production in Ogun State. This is obviously high for a kind of drug that is considered the drug of last resort. However, when compared with other similar studies, it is relatively low. Arena et al. recently revealed high rates of CRE carriage $(28.4 \%)$ among patients from a long-term acute-care rehabilitation facility (LTACRF) in central Italy [13].

Beena et al. reported $66 \%$ of isolates to be carbapenemase producers [12]. Aseem et al. developed the approach to carbapenemase detection in Klebsiella pneumoniae in routine diagnostic laboratories and reported $35.3 \%$ carbapenem resistance in the isolates studied [14]. The prevalence in the current study was also low in comparison with the phenotypic prevalence report of $22.4 \%$ Enterobacteriaceae resistance to third generation cephalosporins in Uganda [15]. In Nigeria, Yusuf et al. reported $33.5 \%$ carbapenemase production in Kano and Kaduna States [16]. However, in a neighboring State, Lagos, Nigeria, Oduyebo et al. determined the prevalence of carbapenem resistance among clinical isolates of Enterobacteriaceae to be $15.2 \%$ [17], a range similar to the present study where our carbapenemase production prevalence is $10.9 \%$. This might be attributed to the absence of a considerable boundary between Lagos and Ogun States, allowing for population interception. Studies carried out around the globe revealed that there was a gradual increase in carbapenem resistance all over the world between 2012 and 2013. For instance, in China, (1.2\%) as reported by Xia et al. [18], EnuguNigeria $(2.5 \%)$ revealed in the study of Ejikeugwu et al. [19], Morocco (2.8\%) reported by Wartiti et al. [20], New Delhi-India (6.9\%) as revealed by Gupta et al. [21] and Taiwan (8.6\%) reported by Lai et al. [22].

Variation in the prevalence rates may be attributed to some factors such as, the varied sampled population, seasonal factors, iatrogenic outcome, carbapenem resistance mechanism sought for in different study and the use and misuse of drugs.

The rate of KPC-producing isolates in the current study was $41.6 \%$ on the average of the total CRE production recorded. This contradicts the studies of Conte et al. and Nagaraj et al. [23, 24]. Zogorianou et al. also reported a very high (97\%, $75 \%$ and $66.4 \%$ ) contribution of blaKPC, $K$. pneumoniae KPC producing Klebsiella pneumoniae isolates respectively to the total burden of CRE [25]. In contrary, Netikul et al. and Ho et al. reported a 
very low incidence of KPC-producing isolates within their CRE isolates [26, 27]. The study of Nayak et al. also reported a slightly low (16.6\%) prevalence of KPC producing Klebsiella pneumoniae as its quota to 54.8\% of the entire CRE in their study [28].

The variation in MHT and CIM result was found among Klebsiella pneumoniae isolates, although, this was not statistically significant $(p=0.005)$.

This present study is in total conformity with the study of Gupta et al. who also reported the same prevalence of $33.3 \%$ as the total contribution of KPC-Klebsiella pneumoniae by modified Hodge's test [21]. This study also agrees with the review of Ssekatawa et al. who highlighted that $\mathrm{CP}$ bacteria have a predominant occurrence in the respiratory tract $(23 \%)$, Blood $(22 \%)$, urinary tract $(19 \%)$ and wounds/pus (18\%) in East Africa [29]. The prevalence of resistance to carbapenem in this study is relatively high, being the last drug of resort and KPC-Kp has a significant contribution to the burden of CRE.

\section{Conclusion}

In conclusion, this study found 110 Enterobacteriaceae isolates from different samples of patients in the intensive care units of two public hospitals in Abeokuta, Ogun State, Nigeria out of which $10.9 \%$ exhibited carbapenem-resistance. Klebsiella pneumoniae carbapenemase-producing Klebsiella pneumoniae prevalence was $3.6 \%$, contributing $33.3 \%$ to the burden of carbapenem production. The occurrence of carbapenem-resistant Enterobacteriaceae in this study was relatively high and KPC-Klebsiella pneumoniae had a significant contribution to the burden of CRE. Hence, a sinewy antibiotic stewardship is needed in this regard.

\section{Conflicts of interest:}

The authors hereby declare that there are no conflicts of interest with respect to this article.

\section{Financial disclosure:}

There is no specific financial interest, relationship and affiliations relevant to the subject of the manuscript.

\section{References}

1-Ambretti, SM, Bassetti P, Clerici P Clerici N

Petrosillo $\mathbf{F}$ Tumietto $\mathbf{P}$, et al. Screening for carriage of carbapenem-resistant Enterobacteriaceae in settings of high endemicity: a position paper from an Italian working group on CRE infections. Antimicrobial Resistance and Infection Control 2019; 8(1): 111.

2-Chotiprasitsakul D, Srichatrapimuk S, Kirdlarp S, Pyden AD, Santanirand P. Epidemiology of carbapenem-resistant Enterobacteriaceae: a 5-year experience at a tertiary care hospital. Infection and Drug Resistance 2019; 12: 461-468.

3-Zheng B, Lv T, Xu H, Yu X, Chen Y, Li J, et al. Discovery and characterisation of an Escherichia coli ST206 strain producing NDM-5 and MCR-1 from a patient with acute diarrhoea in China. International Journal of Antimicrobial Agents 2018; 51(2): 273-275

4-Wang S, Xu L, Chi X, Li Y, Kou Z, Hou P, et al. Emergence of NDM-1- and CTX-M-3Producing Raoultella ornithinolytica in Human Gut Microbiota. Frontiers in Microbiology 2019; 10:2678.

5-Chitnis AS, Caruthers PS, Rao AK, Lamb J, Lurvey R, De Rochars VB, et al. WISE (2012) Outbreak of carbapenem-resistant Enterobacteriaceae at a long-term acute care hospital: Sustained reductions in transmission through active surveillance and targeted interventions. Infection Control and Hospital Epidemiology 33(10):984-992.

6-Temkin E, Adler A, Lerner A, Carmeli Y. Carbapenem-resistant Enterobacteriaceae: biology, epidemiology, and management. Annals of New York Academy of Sciences 2014; 1323:22-42.

7-Khan AU, Maryam L, Zarrilli R. Structure, genetics and worldwide spread of New Delhi Metallo-beta-lactamase (NDM): a threat to public health. BMC Microbiology 2017; 17:101.

8-Nordmann P, Naas T, Poirel L. Global spread of carbapenemase-producing Enterobacteriaceae. 
Emerging Infectious Diseases 2011; 17: 17911798.

9-European Centre for Disease Control and Prevention (ECDC). EARS-NET Report. Surveillance of antimicrobial resistance in Europe 2017. Available at https://ecdc.europa.eu/sites/portal/files/document s/EARS-Net-report-2017-update-jan-2019.pdf

Retrieved on 21 January 2019.

10-Winn W, Allen S, Janda W, Koneman E, Procop G, Schreckenberger P, et al. Introduction. Koneman's Color Atlas and Textbook of Diagnostic Microbiology. Baltimore and Philadelphia: Lippincott Williams \& Wilkins; 2006. p. 21-5

11-Clinical and Laboratory Standards Institute (CLSI) 2017. Performance standards for antimicrobial susceptibility testing CLSI Supplement M100, 27th Edn. Wayne, PA.

12-Beena HB, Shalini SM, Sangeetha J, Ranjeeta A, Bhavana MV. Molecular characterization of extended spectrum $\beta$ lactamase and carbapenemase producing Klebsiella pneumoniae from a tertiary care hospital. Indian Journal of Critical Care Medicine 2019; 23(2): 61-66.

13-Arena F, Vannetti F, Di Pilato V, Fabbri L, Colavecchio OL, Giani T, et al. Diversity of the epidemiology of carbapenemase-producing Enterobacteriaceae in long-term acute care rehabilitation settings from an area of hyperendemicity, and evaluation of an intervention bundle. Journal of Hospital Infection 2018; 100: 29-34.

14-Aseem R, Shenoy S, Mala SS, Baliga S, Ashish A. Approach to carbapenemase detection in Klebsiella pneumoniae in routine diagnostic laboratories. Journal of Clinical and Diagnostic Research 2016; 10(12): DC24DC27.
15-Okoche D, Asiimwe BB, Katabazi FA, Kato

L, Najjuka CF. Prevalence and characterization of carbapenem-resistant Enterobacteriaceae isolated from Mulago National Referral Hospital, Uganda. PLoS ONE 2015; 10(8): e0135745.

16-Yusuf I, Yusha'u M, Sharif AA, Getso MI, Yahaya H, Bala JA Detection of Metallobetalactamases among gram negative bacterial isolates from Murtala Muhammad Specialist Hospital, Kano and Almadina Hospital Kaduna, Nigeria. Bayero Journal of Pure and Applied Sciences 2012; 5(2): 84-88.

17-Oduyebo OO, Falayi OM, Oshun P, Ettu AO. Phenotypic determination of carbapenemase producing Enterobacteriaceae isolates from clinical specimens at a tertiary hospital in Lagos, Nigeria. The Nigerian Postgraduate Medical Journal 2015; 22:223227.

18-Xia Y, Liang $\mathbf{Z}, \quad$ Su $\quad X$, Xiong Y. Characterization of carbapenemase genes in Enterobacteriaceae species exhibiting decreased susceptibility to carbapenems in a University hospital in Chongqing, China. Annals of Laboratory Medicine 2012; 32:270-275.

19-Ejikeugwu PC, Ugwu CM, Araka CO, GUGU TH, Iroha IR, Adikwu MU, et al. Imipenem and meropenem resistance among ESBL producing Escherichia coli and Klebsiella pneumoniae clinical isolates. International Research Journal of Microbiology 2012; 3(10): 339-344.

20-ELWartiti MA, Bahmani FZ, Elouennass M, Benouda A. Prevalence of carbapenemase producing Enterobacteriaceae in a university hospital in Morocco: A 19 months prospective study. International Arabic Journal of Antimicrobial Agents 2012; 2:372-377. 
21-Gupta V, Bansal N, Singla N, Chander

J. Occurrence and Infection phenotypic detection of class A carbapenemases among Escherichia coli and Klebsiella pneumoniae blood isolates at a tertiary care center. Journal of Microbiology Immunology and Infection 2013; 46:104-108.

22-Lai CC, Wu UI, Wang JT, Chang SC. Prevalence of carbapenemase-producing Enterobacteriaceae and its impact on clinical outcomes at a teaching hospital. Journal of the Formosan Medical Association 2013; 112:492496.

23-Conte V, Monaco M, Giani T, D'Ancona F, Moro ML, Arena F, et al. Molecular epidemiology of KPC-producing Klebsiella pneumoniae from invasive infections in Italy: Increasing diversity with predominance of the ST512 clade II sublineage. Journal of Antimicrobial Chemotherapy 2016; 71(12):3386-3391.

24-Nagaraj S, Chandran SP, Shamanna P, Macaden R. Carbapenem resistance among Escherichia coli and Klebsiella pneumoniae in a tertiary care hospital, South India. Indian Journal of Medical Microbiology 2012; 30: 9395.

\section{5-Zagorianou A, Sianou E, Iosifidis E, Dimou} V, Protonotariou E, Miyakis S, et al. Microbiological and molecular characteristics of carbapenemase producing Klebsiella pneumoniae endemic in a tertiary Greek hospital during 2004-2010. Eurosurveillance 2012; 17(7):12-18.
26-Netikul T, Kiratisin P. Genetic characterization of carbapenem-resistant Enterobacteriaceae and the spread of carbapenem-resistant Klebsiella pneumoniae ST340 at a university hospital in Thailand. PLoS One 2015; 10(9): e0139118.

27-Ho PL, Cheung YY, Wang Y, Lo WU, Lai EL, Chow KH, et al. Characterization of carbapenem-resistant Escherichia coli and Klebsiella pneumoniae from a healthcare region in Hong Kong. European Journal of Clinical Microbiology and Infectious Diseases 2016; 35(3):379-385.

28-Nayak S, Singh S, Jankhwala S, Pradhan R. Prevalence, characterization and clinical significance of Klebsiella pneumoniae carbapenemase (KPC) producing Klebsiella pneumoniae. International Journal of Medical Research and Health Sciences 2014; 3(4):797803.

29-Ssekatawa K, Byarugaba DK, Wampande E, Ejobi F. A systematic review: the current status of carbapenem resistance in East Africa. BMC Research Notes 2018; 11:629

Ojo AE, Adebajo SO, Fasina KA, Odejinmi B, Ojo OA, Ooladotun A, Ibeaha J. Phenotypic detection of carbapenem resistant Enterobacteriaceae and characterization of Klebsiella pneumoniae carbapenemase (KPC) producing Klebsiella pneumoniae. Microbes Infect Dis 2021; 2(4): 760-766. 